\title{
In-Line Electromagnetic Actuator for Fuel Modulation
}

\author{
Robert S. Okojie ${ }^{1}$, Carl W. Chang ${ }^{2}$, Roger D. Meredith ${ }^{3}$, Randy Thomas ${ }^{4}$, George Kopasakis ${ }^{5}$ \\ 1,3,4,5 NASA Glenn Research Center, Cleveland, OH 44135 USA \\ ${ }^{2}$ Vantage Partners, LLC, NASA Glenn Research Center, Cleveland, OH 44135 USA
}

\begin{abstract}
An in-line electromagnetic actuator with normally-open configuration has been developed for the purpose of exploring its application as a fuel modulator for the active control of combustor thermo-acoustic instabilities. The actuator is based on the spring-coil-plunger mechanism with the plunger designed to be suspended by crossed cantilever beam springs. Operational specification was set for a 1000 psi maximum inlet fuel pressure and modulation for a broad frequency bandwidth that encompasses frequencies that are typically associated with combustor thermo-acoustic instabilities. Various test results demonstrated fuel modulation up to $1200 \mathrm{~Hz}$ for coil excitation voltage between 2.3 and $4.6 \mathrm{~V}$ and current between 16 and $48 \mathrm{~mA}$, respectively. The initial goal of this work was to demonstrate fuel modulation based on the concept of a normally-open fuel modulator with tethered plunger displacement.
\end{abstract}

\section{Introduction}

The demand for lower emissions (LE) in aircraft gas-turbine engines has resulted in advanced combustor designs that are critically dependent on lean-burning (LB) operation. However, LB/LE combustors are susceptible to thermoacoustic instabilities that can produce large pressure oscillations within the combustor. This can at a minimum disrupt compressor flow or potentially lead to premature mechanical failures. A combustor is essentially an acoustic resonator. As an engine is run through its operating range, there are states where the heat release coupling with the acoustics forms unacceptably high pressure oscillations. Such instances have been well documented and reported [1,2]. This problem is expected to be more pronounced in LB/LE combustion systems because less system damping is present with LB combustors due to reduced liner cooling [3] or because of the higher volumetric heat release of these types of combustors. Traditionally this kind of problem is resolved with pre-programmed open-loop adjustments; however, there is no guarantee that such techniques can deal with all of the instabilities. Previous works to study combustor thermo-acoustic instabilities have relied upon feedback signals from pressure sensors [4]. This feedback signal is now being utilized in conjunction with fuel modulating actuators to actively control the instabilities, which has led to extensive active research in this area $[5,6]$.

A literature review of actuators for fuel modulation showed two prominent actuation principles were applied, one being electromagnetic and the other piezoelectric. While piezoelectric actuators have the advantages of generating large forces and fast response times, they require relatively high voltages for actuation. Also, their displacement reduces significantly with increased temperature. A solenoid configured electromagnetic actuators was chosen in this development because it offers the advantage of a lower drive voltage, and suffers less from reduced displacement with increasing temperature compared to a piezoelectric actuators [7].

Most fuel actuators found in automobiles are normally-closed prior to excitation by a spring pre-load mechanism. In this work, a normally-open In-Line Electromagnetic (ILEM) actuator with a tethered spring-plunger system was

\footnotetext{
${ }^{1}$ Research Electronics Engineer, Smart Sensors \& Electronics Systems Branch, Member AIAA.

${ }^{2}$ Research Electronics Engineer, Smart Sensors \& Electronics Systems Branch.

${ }^{3}$ Electronics Engineer, Smart Sensors \& Electronics Systems Branch.

${ }^{4}$ Electronics Engineer, Intelligent Control and Autonomy Branch.

${ }^{5}$ Research Electronics Engineer, Intelligent Control and Autonomy Branch, Member AIAA
} 
conceptualized, developed, and tested for fuel modulation in gas turbine engines. Modulation frequencies as high as $1.2 \mathrm{kHz}$ with input fuel pressures of approximately 300 psia was demonstrated. In this development the ILEM modulator in conjunction with the silicon carbide high temperature pressure sensors [4] is described with the future goal of using them to demonstrate closed loop active control of combustion instabilities in gas turbine engines.

\section{In-Line Electromagnetic Fuel Modulator Description}

The structure of the ILEM that was developed in this effort is shown in Figure 1. It consists of the following low carbon ferromagnetic components: the housing (sleeve), top cap, bottom cap, and a central sliding plunger within a ceramic bobbin. The coil wound was made of ceramic coated nickel wire and wound around the ceramic bobbin. The plunger is tethered and suspended at one end (near the top cap) by a 4-cantilever non-magnetic stainless steel beamspring system. The spring system acts to restore the plunger to its initial normally-open position when the coil is deenergized. Fuel flows in through the opening in the top cap and flows around the central plunger through the central hole in the bobbin, and then exits through the bottom cap. The function of the design is best modeled as a magnetic circuit. When electrical current is applied to the magnetic coil, the resulting magnetic field induces a magnetic flux.

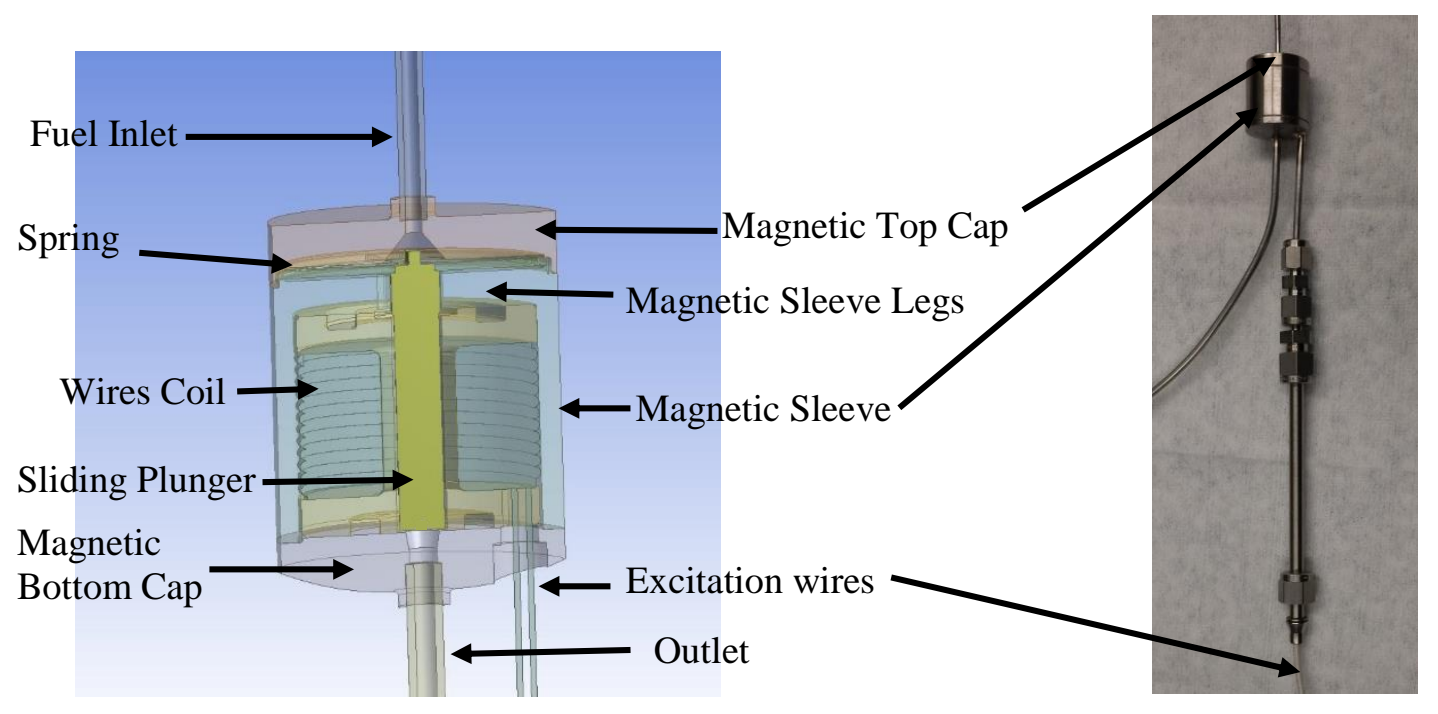

(a)

(b)

Fig. 1 Cross sectional image of ILEM modulator (a). Sliding plunger is pulled towards the magnetic bottom cap when electrical current is applied to the magnetic coil, closing the gap between the tip of the plunger and the bottom cap. Photograph of actual ILEM modulator (b).

The tendency of the magnetic flux is to flow through the least magnetic resistance or the least reluctance path, which minimizes the energy in such a magnetic circuit. In this design the path of least reluctance is through the ferromagnetic parts that consist, of the plunger, bottom cap, and magnetic housing (the magnetic sleeve with legs). The air gap between the tip of the sliding plunger and the bottom cap results in a higher reluctance. As the plunger is free to move closer to the bottom cap thereby reducing this air gap distance, a magnetomotive force between the tip of plunger and the bottom cap is generated, which serves to minimize this air gap and to reduce the energy stored in the magnetic circuit. As the plunger is actuated the cone-shaped tip moves downward to close the gap between the tip of the plunger and the magnetic bottom cap thereby, controlling fuel flow. Intermittently energizing the coil, would then result in the modulation of the fuel.

The position of the magnetic plunger is dependent on the strength of the magnetomotive force driving the magnetic circuit. A stronger generated magnetic flux will result in a larger force on the plunger pulling it closer to the bottom cap, thereby closing the fuel flow path further. The magnetomotive force applied to the magnetic circuit is directly related to the electrical current applied to the magnetic coil. Therefore, increasing the electrical current applied to the magnetic coil will result in increasing closing force on the plunger. However, once saturation of the magnetic components occurs, the rate of increase of the magnetic force compared to the applied electrical current will drop to a 
much lower value. Therefore, operation of the magnetically actuated valve is generally confined to the region of operation prior to magnetic saturation.

\section{Test Setup}

The prototype actuator was tested using jet fuel in a bench top setting. The test setup with the actuator is shown in Figures 2 (a) and (b) for the diagram and physical setup, respectively. The fuel accumulator is placed ahead of the actuator to filter fuel pressure fluctuations on the supply side and provide a more consistent fuel pressure to the inlet. Pressure sensors are placed at multiple positions in the test setup to measure both the static and dynamic pressures as shown in Figure 2 (a). A needle valve is placed after the actuator to simulate the injector and to allow varying the orifice size in order to vary its flow number (FN).

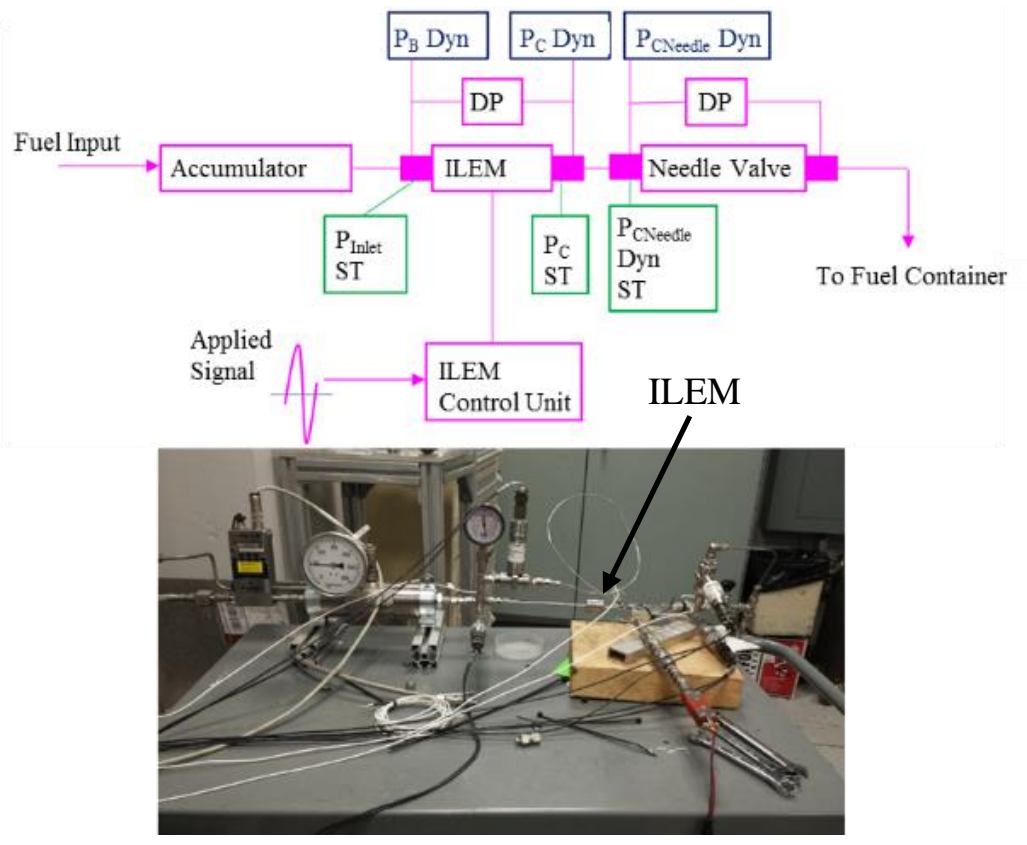

Fig. 2 Fuel test circuit. Diagram of circuit with pressure sensors shown (above). Photograph of actual test circuit with ILEM modulator shown (below).

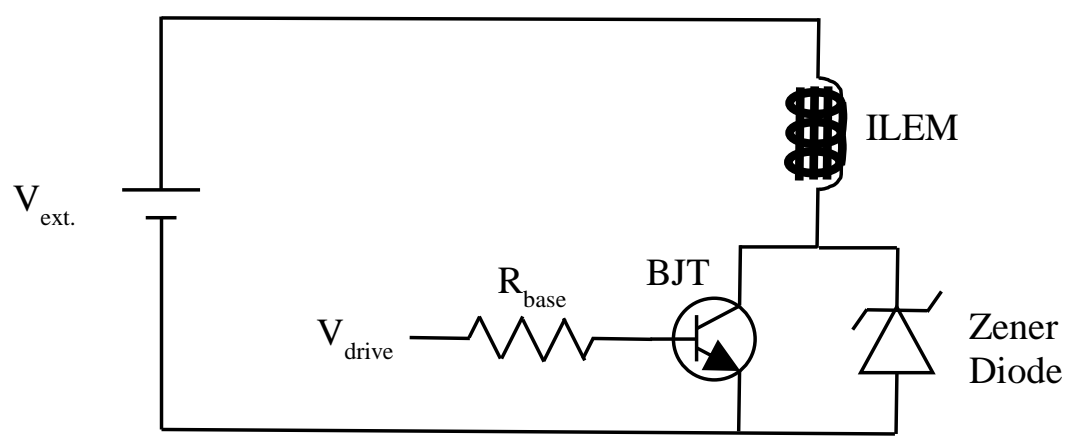

Fig. 3 ILEM control unit circuit. Circuit consists of a BJT, Zener diode, base resistor to limit base current to the BJT, $V_{\text {ext }}$ supply voltage, and $V_{\text {drive }}$ to drive the circuit. The magnetic coil is driven and has current flowing through it when the BJT is turned on.

The magnetic coil is driven by the ILEM control unit which uses the circuit shown in Figure 3. The circuit consists of a power bipolar junction transistor (BJT) driving current through the magnetic coil. A Zener diode is also present in the circuit to bypass all current through it when the voltage to the coil is switched off. Since the current in the coil (an inductor) momentarily remains constant while the transistor is turned off, a kickback voltage across the coil results. 
Thus, the use of the Zener diode in the circuit prevents damage to the rest of the circuit components. The base of the transistor is fed a time-varying voltage $\left(\mathrm{V}_{\text {drive }}\right)$ to turn the transistor on and off, while a low voltage power supply $\left(\mathrm{V}_{\text {ext }}\right)$ is used to supply the voltage and current to the circuit.

\section{Test Conditions and Results}

The ILEM modulator was tested using jet fuel in order to determine its effectiveness in modulating fuel flow. Inlet pressure for the ILEM modulator was maintained at approximately $300 \mathrm{psi}$, with a flow rate of approximately 43 pounds per hour (PPH). The power supply voltage to the ILEM control unit circuit was set at $\mathrm{V}_{\mathrm{ext}}=4 \mathrm{~V}$, and the drive voltage $\mathrm{V}_{\text {drive }}$ was $8 \mathrm{~V}$ peak-to-peak. Under these conditions, measurements were taken of the pressures at the sensor locations shown in Fig. 2. Prior to the ILEM modulator being turned on and driven at the various excitation frequencies, two steady state measurements were performed with all drive electronics turned off. Following the two steady state measurements, the ILEM modulator was driven with a sinusoidal voltage applied to the base of the BJT at discrete frequencies from $100 \mathrm{~Hz}$ up to $1200 \mathrm{~Hz}$ and back down to $100 \mathrm{~Hz}$ in $100 \mathrm{~Hz}$ increments as fuel flowed through it. Pressure measurements were taken at each of these frequencies for the given flow rate and pressure indicated above. Results of the steady state measurements as well as the measurements from $100 \mathrm{~Hz}$ to $1200 \mathrm{~Hz}$ are shown in Figure 4, which plots the spectral density in psi/Hz of the $\mathrm{P}_{\mathrm{C} \_}$Dyn pressure measurement.

\begin{tabular}{|c|c|c|c|c|c|}
\hline Frequency $(\mathrm{Hz}) \uparrow$ & $\mathbf{P}_{\mathrm{C}}$ Dyn (PSI) & $\mathbf{P}_{\text {CNeedle }}$ Dyn & $\begin{array}{l}\text { Frequency } \\
(\mathrm{Hz}) \downarrow\end{array}$ & $\mathbf{P}_{\mathrm{C}}$ Dyn (PSI) & $\mathbf{P}_{\text {CNeedle }}$ Dyn \\
\hline 100 & 3.26 & NA & 100 & NA & NA \\
\hline 200 & NA & 0.71 & 200 & NA & 0.93 \\
\hline 600 & 0.49 & NA & 600 & 0.60 & NA \\
\hline 700 & 6.81 & $\mathrm{NA}$ & 700 & 8.28 & NA \\
\hline 800 & 3.01 & NA & 800 & 3.08 & NA \\
\hline 900 & 3.6 & 0.45 & 900 & 4.10 & 0.46 \\
\hline 1000 & 2.2 & 0.63 & 1000 & 2.57 & 0.64 \\
\hline 1100 & 1.45 & 1.29 & 1100 & 1.53 & 1.45 \\
\hline 1200 & 1.16 & NA & 1200 & 1.16 & NA \\
\hline
\end{tabular}

Table 1 Maximum measured pressure change at $P_{C}$ Dyn for each frequency of operation. Table includes data measured as frequency was increased and also as it was decreased. Note values labeled NA were the result of the measured maximum pressure change being recorded at a different frequency than the driven frequency listed.

Table 1 summarizes the maximum spectral amplitude of the pressure modulation measured for $\mathrm{P}_{\mathrm{C} \_}$Dyn and $\mathrm{P}_{\mathrm{CNeedle} \_}$Dyn when for the drive voltage and the frequencies described above. Values for $\mathrm{P}_{\mathrm{C} \_}$Dyn and $\mathrm{P}_{\mathrm{CNeedle} \_D y n}$ are marked NA for several entries due to the mismatch between the drive frequency and the frequencies at which the measured maximum value in the frequency domain occurred. It should be noted that the maximum recorded

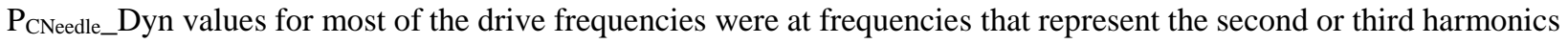
of the drive frequency and were therefore marked as NA. Reliable maximum recorded values of $\mathrm{P}_{\mathrm{CNeedle}}$ Dyn that matched the drive frequency occurred at the frequencies of $200 \mathrm{~Hz}$ and 900 to $1100 \mathrm{~Hz}$. Values for both P $\mathrm{P}_{\mathbf{C}}$ Dyn and

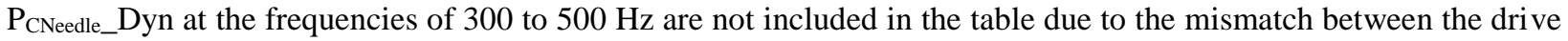
frequency and the frequency at which the maximum values were recorded. The cause of the harmonics at these frequencies are yet to be identified. The possibility of magnetic after-effect could not be ruled out [8]. However, it should be noted that the valve designed FN is considerably higher than that that can be obtain from the Needle valve 
due to facility flow limitations, and for such conditions the pressure modulation out of the valve will be compromised. Conditions where the valve FN is equal to or a little less than that of the needle valve would be expected to produce the best conditions for valve modulation. The largest values of $\mathrm{P}_{\mathrm{C} \_}$Dyn occurred at the frequency range of 700 to 900 $\mathrm{Hz}$, where a maximum value of 8.28 PSI was recorded at $700 \mathrm{~Hz}$. The largest value recorded for $\mathrm{P}_{\mathrm{CNeedle} \_}$Dyn occurred at $1100 \mathrm{~Hz}$ and was only slightly less than the value of $\mathrm{P}_{\mathrm{C} \_}$Dyn at this frequency.

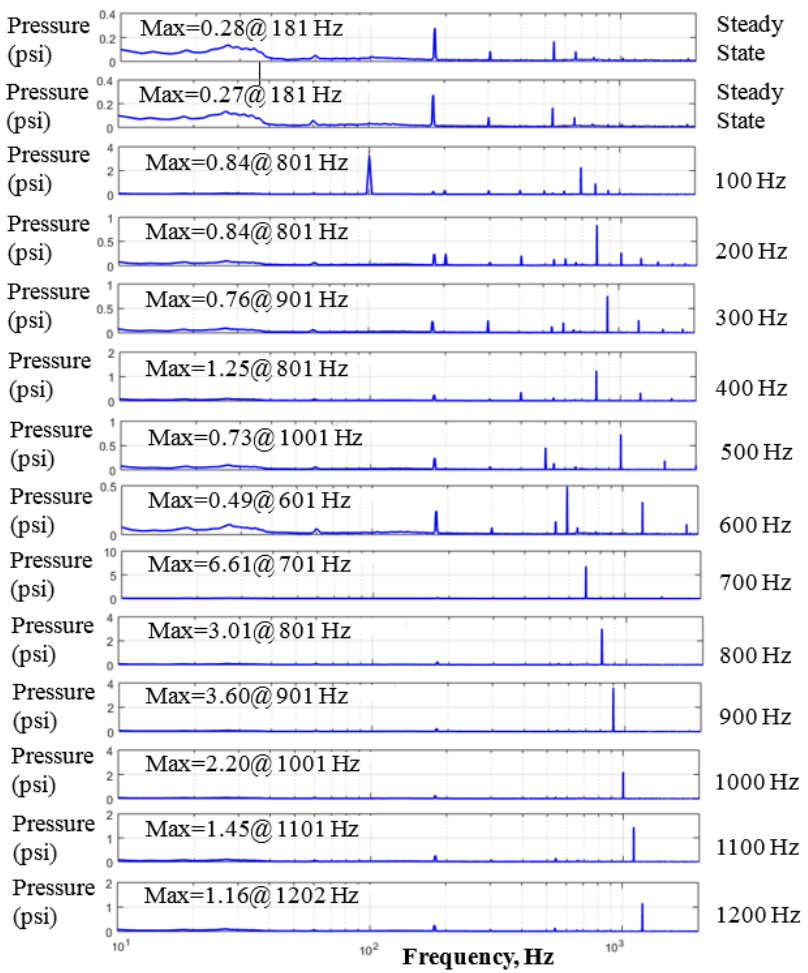

Fig. 4 Measurement results in frequency domain for the ILEM modulator with flow conditions of 300 PSI and 43 PPH flow rate. $V_{\text {ext }}$ on ILEM control unit at $4 \mathrm{~V}$, and $V_{\text {drive }}$ at $8 \mathrm{~V}$ peak-to-peak. Pc dyn pressure plotted vs frequency as ILEM modulator is at steady state (SS) and from $100 \mathrm{~Hz}$ to $1200 \mathrm{~Hz}$ in $100 \mathrm{~Hz}$ increments. The ILEM modulator FN was $\sim 15.0$, and the needle valve FN was $\sim 2.50$

The excitation voltage of the actuator was increased in order to increase the current flowing through the coil, hence, increase the magnetic flux in order to increase the plunger stroke and allow it to come closer to the bottom cap. This was done with the goal of constricting the flow and produce higher pressure at the actuator output. The ILEM modulator was driven with a $\mathrm{V}_{\text {ext }}$ of $4.5 \mathrm{~V}$, and a $\mathrm{V}_{\text {drive }}$ of $8 \mathrm{~V}$ peak-to-peak at approximately the same inlet pressure and flow rate described above. It was found that at lower frequencies, the plunger had momentarily came in contact or near contact with the bottom cap in a phenomenon described as electromagnetic snap-in. Just prior to the onset of snap-in, the input pressure increases and the flow rate decreases or stops. Snap-in occurs when the magnetic force pulling the plunger closer to the bottom cap exceeds the restoring force from the 4-beam tethered spring that is used to return the plunger to its original position and any force exerted on the plunger from the fluid. The magnetic force is a nonlinear force that increases rapidly with decreasing gap between the plunger and bottom cap, as the force is inversely proportional to the square of the gap between the plunger and bottom cap. The restoring force from the spring has a linear relationship to the displacement of the plunger. Therefore, at a certain gap the magnetic force will exceed the restoring force of the spring and then the magnetic force rapidly increases as the gap distance decreases, thereby causing the plunger tip to snap-in to the bottom cap. When this occurs, the plunger and bottom cap are in near or intimate contact, which significantly constricts the fluid flow through the ILEM modulator. Indeed this phenomenon was observed when the ILEM modulator was driven at $100 \mathrm{~Hz}$ as shown in Figure 5. As can be seen in Figure 5, the pressure drop across the ILEM modulator (ILEM DP) increases rapidly from near zero to over 400 PSI as snap-in occurs while a large drop in the flow rate from $42 \mathrm{pph}$ to about $24 \mathrm{pph}$ was also observed. The modulator and needle 
flow numbers was also affected as they dropped from 16 to 1 and 2.5 to 1 , respectively. The reason the ILEM DP exceeded the inlet pressure at that point is because the facility regulator is no longer able to regulate the facility fuel pressure when the flow restriction exceeds a certain limit.

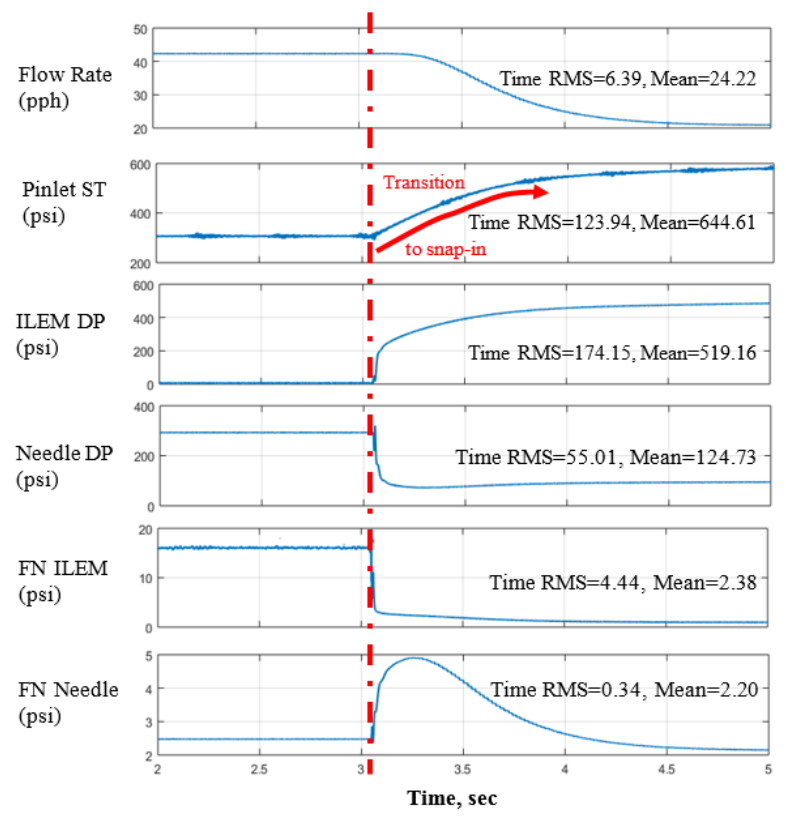

Fig. 5 Time domain plot of measurements of pressures, differential pressures (DP), and flow numbers as the ILEM modulator was operated at $100 \mathrm{~Hz}$. The ILEM modulator was driven at $100 \mathrm{~Hz}$, with a $\mathrm{V}_{\mathrm{ext}}=4.5 \mathrm{~V}$. Inlet pressure to the ILEM modulator started at 300 PSI and as shown at the dotted red line began to increase as the transition to snap-in occurred. After snap-in the pressure rose to 645 PSI. Similarly the flow rate began at $\sim 42$ PPH and dropped to 24 PPH after snap-in of the ILEM.

\section{Discussion}

The initial primary goal of this work was to demonstrate the functional capabilities of this prototype ILEM actuator for its ability to modulate fuel flow at various frequencies. The results of benchtop testing described above have shown that the ILEM modulator is capable of modulating fuel flow at frequencies of approximately $600 \mathrm{~Hz}$ up to $1200 \mathrm{~Hz}$. However, at frequencies below $600 \mathrm{~Hz}$ the ILEM modulator did not provide sufficient modulation at the drive frequency. The measured data at the lower frequencies indicated that the strength of the modulation was not sufficient to overcome the background noise in the system. This noise was more prevalent at a frequency of $180 \mathrm{~Hz}$, and it was showing in the data especially, for the test frequencies below $600 \mathrm{~Hz}$. The source of this noise has not been determined, but it's assumed to be coupling noise from the grid power ( $3^{\text {rd }}$ harmonic); coupling through the non-transformer isolated drive circuit of the modulator.

A second consideration, not yet fully evaluated, is the optimum settings for the ILEM control unit circuit. Test results presented here were taken with conservative drive voltages applied to the ILEM control unit circuit, in order to ensure that a complete set of data could be captured in the limited time available for the benchtop test. Initial explorations of higher drive voltages seem to indicate that the full capability of the ILEM modulator has yet to be untapped in regards to achieving higher modulated pressure drops. For example, larger differential pressure was obtained at the actuator outlet ( $\mathrm{P}_{\mathrm{C}-\mathrm{Dyn}}$ ) when the drive voltage was pre-biased with a $3 \mathrm{VDC}$ before the sinusoidal drive voltage was applied. This was the result of plunger being pulled down toward the bottom cap prior to actuation. Further exploration and optimization of the drive voltages applied to the ILEM control unit are necessary to fully optimize the resulting modulation capabilities of the ILEM modulator, especially at lower frequencies. 
A final consideration is the snap-in phenomenon that is present at very low drive frequencies. This phenomenon should be controllable with a simple modification to the plunger and spring. The addition of a nonmagnetic spacer to the tip of the plunger would prevent it from making full contact with the top cap and thereby prevent the snap-in phenomenon while at the same time provide higher pressure drops. A secondary consideration of the snap-in phenomenon is that the snap-in phenomenon test results indicates that the ILEM modulator is tunable for different flow numbers. By applying a constant DC current to the magnetic coil through the control unit, the plunger DC position can be changed, which creates a smaller average gap with the bottom cap, thereby changing the flow number of the ILEM modulator. This is another capability that has yet to be fully explored for the ILEM modulator functionality.

\section{Conclusion}

In this report an Electromagnetic fuel modulator design is demonstrated that is capable of providing pressure modulations at frequencies from 600 to $1200 \mathrm{~Hz}$. For this first generation ILEM fuel modulator, the results indicated that with an excitation voltage of $4.0 \mathrm{~V}$ and driver magnitude of $8 \mathrm{~V}_{\mathrm{p}-\mathrm{p}}$, the ILEM was capable of modulating fuel at its outlet up to $1.2 \mathrm{kHz}$, with the best response occurring near 600-700 Hz. Future work will include improvements to the drive voltage levels of the control unit circuit and/or changes to modulator design itself in order to optimize performance. This will include finding optimum drive voltages to improve the pressure change the modulator is capable of. In addition, future work will also entail fully exploring the capabilities of tuning the flow number of the modulator by applying DC current to bias the set point of the plunger in the modulator. Finally, work to add a nonmagnetic spacer to the tip of the plunger of the modulator will be explored in order to prevent snap-in at lower frequency operation of the modulator.

\section{Acknowledgments}

This work was performed under the Active Combustion Control task of the NASA Transformational Tools and Technologies project of the Aeronautics Research Mission Directorate. We thank the technical staff of the Combustion Branch for their support in preparing the benchtop test stand.

\section{References}

1. Fleifil, M., Annaswamy, A. M., Ghoneim. Z. A., and Ghoniem, A. F., "Response of a Laminar Premixed Flame to Flow Oscillations: A Kinematic Model and Thermoacoustic Instability Results," Combustion and Flame Vol. 106, 1996, pp. 487510.

2. Culick, F. E. C., "Some Recent Results for Nonlinear Acoustics in Combustion Chambers,"AIAA Journal, Vol. 32(1), 1994, pp. 146-169.

3. Lee, J. G. and Santavicca, D. A., "Experimental Diagnostics for the Study of Combustion Instabilities in Lean Premixed Combustors," Journal of Propulsion and Power, Vol. 19, No. 5, 2003, pp. 735-750.

4. Okojie, R. S., DeLaat, J. C., Saus, J. R., "SiC Pressure Sensor for Detection of Combustor Thermoacoustic Instabilities [Aircraft Engine Applications]," The 13 $3^{\text {th }}$ International Conference on Solid-State Sensors, Actuators and Microsystems, 2005. Digest of Technical Papers, Vol. 1, 2005, pp. $470-473$.

5. DeLaat, J. C.; Kopasakis, G.; Saus, J. R.; Chang, C. T; Wey, C; “Active Combustion Control for a Low-Emissions Aircraft Engine Combustor Prototype - Experimental Results,” AIAA-2012-783.

6. Kopasakis, G., DeLaat, J. C., Chang, C. T., "Adaptive Instability Suppression Controls Method for Aircraft Gas Turbine Engine Combustors," Journal of Propulsion and Power, Vol.25, 2009, pp. 618-627.

7. Lu, F. K. and Jensen, D.S., "Potential Viability of a Fast-Acting Micro-Solenoid Valve for Pulsed Detonation Fuel Injection," 41st Aerospace Sciences Meeting and Exhibit, 6-9 January 2003, Reno, Nevada, AIAA 2003-888.

8. Ando, R, Koizumi, M, and Ishikawa, T, "Development of a Simulation Method for Dynamic Characteristics of Fuel Injector," IEEE Transactions on Magnetics, Vol. 37 (5), 2001, pp. 3715. 